\title{
Recombination of Intersecting D-branes by Local Tachyon Condensation
}

\author{
Koji Hashimoto and Satoshi Nagaoka \\ Institute of Physics, University of Tokyo \\ Komaba, Tokyo 153-8902, Japan \\ E-mail: koji@hep1.c.u-tokyo.ac.jp, nagaoka@hep1.c.u-tokyo.ac.jp
}

\begin{abstract}
We provide a simple low energy description of recombination of intersecting D-branes using super Yang-Mills theory. The recombination is realized by condensation of an off-diagonal tachyonic fluctuation localized at the intersecting point. The recombination process is equivalent to brane-antibrane annihilation, thus our result confirms Sen's conjecture on tachyon condensation, although we work in the super Yang-Mills theory whose energy scale is much lower than $\alpha^{\prime}$. We also discuss the decay width of non-parallelly separated D-branes.
\end{abstract}

Keywords: D-branes, Tachyon Condensation. 


\section{Contents}

1. Introduction 1

2. D-brane recombination realized in Yang-Mills 3

2.1 Fluctuation analysis of Yang-Mills

2.2 Realization of D-string recombination 6

3. Large intersection angle and Sen's conjecture 9

4. Dynamical aspects of recombination: decay width 13

5. Future directions

A. Higher order in $\theta$ and NBI corrections 18

B. Tachyon mass squared to higher order in $\phi$

\section{Introduction}

The significance of D-brane dynamics in string theories has been widely recognized, and for unified formulation of nonperturbative string/M theory it is believed to be promising to adopt D-branes as fundamental objects. Low dimensional D-branes, such as D-particles and D-instantons, have been utilized along this direction [1], while higher dimensional D-branes have relative difficulty due to the complicated interaction of the extended worldvolumes. One of such interaction is the recombination, or equivalently reconnection, of intersecting D-branes (especially D-strings). For fundamental strings, the recombination effect has been introduced as an essential interaction in an open string field theory [2]. It strongly suggests that the recombination is one of the most important mechanisms to be clarified, for formulating any field theory of interacting D-branes. On the other hand, recombination of Dbranes turned out to be an intriguing realization of Higgs phenomena in Standard Model on intersecting branes [3] and inflation in braneworld scenario [4]. The recombination appears as an essential dynamics in these recent developments in string phenomenology, hence its precise formulation in string theory is required.

It is well-known [5] that the recombination is due to the tachyon mode appearing as the lowest mode of a string connecting the intersecting D-branes (see Fig. (1)), 
although the mechanism has not been shown explicitly so far. As seen below, this is related to Sen's conjectures [6] stating that tachyon condensation on brane-antibrane is equivalent to the disappearance of them. When the intersection angle $\theta$ is $\pi$, the intersecting D-branes are just a parallel brane-antibrane pair. The relevant tachyon mode appears in excitation of a string connecting the two. If we decrease $\theta$ a little, then this tachyonic mode is localized near the intersection point. Provided the tachyon condensation makes the branes disappear, it affects them only locally and the branes are recombined (See Fig. 4). The verification of the Sen's conjectures, especially on the disappearance of open string degrees of freedom, has not been shown in a complete form yet. So it would be possible to say that showing the D-brane recombination explicitly is qualitatively equivalent to the verification of the Sen's conjectures. In addition, the system of intersecting D-branes includes the parallel brane-antibrane system as a special limit $\theta=\pi$, and naively it is expected that any multi-body systems of D-branes are involved with intersecting branes generically, rather than its special case $\theta=\pi$ of the parallel brane-antibrane. This leads us to motivate the study of the local tachyon condensation on the intersecting branes, which is the recombination process.

In this paper, we provide a very simple description of the recombination of intersecting D-branes by analysing corresponding background in super Yang-Mills theories which are low energy effective theories of D-branes. We identify one of the fluctuation eigen modes with the tachyon state concerning the annihilation of branes, and show that the condensation of this mode exactly gives the recombination of the intersecting branes. Our description provides a geometrical realization of the open string tachyon condensation.

In Ref. [7], fluctuation spectra of Yang-Mills theory have been studied for a Tdualized configuration of the intersecting branes wound on a torus. The authors of Ref. [7] showed the agreement between the Yang-Mills fluctuation spectra and string theory spectra, while due to the T-duality geometrical picture of the recombination was difficult to be seen. Our work does not involve the T-duality and fills the gap.

In Section 2 we study a $1+1$ dimensional $S U(2)$ super Yang-Mills theory which is induced on parallel D-strings at low energy.* We turn on a linear Higgs background in the diagonal component, which amounts to turning on the intersecting angle $\theta$. The fluctuation analysis around this background shows that there appears a tachyonic mode whose mass squared (and its dependence on $\theta$ ) coincides with the worldsheet result of Ref. [8], for small $\theta$. After diagonalizing the fluctuation, we see that the tachyonic mode is exactly associated with the brane recombination. All the computations are carried out in the low energy approximation of string theory whose energy scale is much lower than the string scale $\alpha^{\prime}$. This is possible since at small $\theta$ the tachyon becomes almost massless.

*Higher dimensional D-branes intersecting with a single angular parameter can be treated with a trivial generalization of our work. 
We also attempt to analyse the behavior of $\theta \sim \pi$, in Section 3. In the limit $\theta=\pi$, the brane configuration becomes a parallel coincident D1-antiD1 pair. We first clarify the relation between the D-brane recombination and the Sen's conjectures on the tachyon condensation on brane-antibrane. With natural ansatz from string scattering amplitudes, we adopt a tachyon action and its coupling to the gauge fields living on the D-branes. This natural tachyon action is used to show that the worldsheet spectrum near $\theta=\pi$ is precisely reproduced in the fluctuation analysis. Furthermore, the geometrical recombination is shown also in this system $\theta \sim \pi$ by analysing the backreaction to the transverse scalar field.

In Section 4, we describe dynamical aspects of the recombination by evaluating the decay width of non-parallelly separated D-branes. We show that for smaller intersection angle $\theta$, the decay probability gets smaller and the system approaches a supersymmetric system of parallel D-branes. We speculate on how the recombination process proceeds.

The low energy Yang-Mills approximation in Section 2 is valid for small intersection angle $|\theta| \ll 1$, so in Appendix A we include the non-Abelian Born-Infeld (NBI) corrections to it, to see how the string spectrum with the higher order in $\theta$ is reproduced in the fluctuation spectrum. We use $\left(\alpha^{\prime}\right)^{2} F^{4}$ corrections in the NBI [9] and follow the techniques developed in Ref. [7], to find complete agreement in the spectra to the order $\theta^{3}$.

\section{D-brane recombination realized in Yang-Mills}

Parallelly placed multiple D-branes in type II superstring theories have super YangMills theory as their low energy description [10]. The non-Abelian structure stems from excitations of strings connecting different D-branes (see Fig. 1). When an intersection angle $\theta(0<\theta<\pi)$ is turned on, these strings get confined to the intersection point so that the energy coming from the tension times its length is minimized. A part of the spectrum of the Neveu-Schwarz sector of this localized open strings was studied in Ref. [8, 11] giving

$$
m^{2}=\left(n-\frac{1}{2}\right) \frac{\theta}{\pi \alpha^{\prime}},
$$

where $n(\geq 0)$ is an integer, and $\theta$ is the intersection angle $(\theta=0$ corresponds to a coincident parallel D-branes, see Fig. 1). ${ }^{\dagger}$ In this paper we treat the simplest case of turning on only a single intersection angle, while generically with higher dimensional D-branes we may consider more parameters for the angles.

The important observation of Eq. (2.1) is that for $n=0$ the mass squared becomes negative, and this is the tachyonic mode of our concern. Since naively the

${ }^{\dagger}$ Ref. [8] studied also the Yang-Mills viewpoint. 




Figure 1: Two D-strings are intersecting with each other at an angle $\theta$. The wavy line depicts a fundamental string connecting the D-strings. The worldvolumes are parametrized by the horizontal direction $x$ while its displacement from the $x$ axis is given by the transverse Higgs field $Y(x)$.

intersecting branes can be recombined and lower the total energy by shortening their lengths, it has been expected that the tachyonic mode $n=0$ is an indication of this recombination [5]. To show this mechanism explicitly is our aim of this paper.

\subsection{Fluctuation analysis of Yang-Mills}

We start with a $1+1$ dimensional $S U(2)$ Yang-Mills action with maximal supersymmetries, ${ }^{\ddagger}$

$$
S=-\operatorname{Tr} \int d t d x^{1}\left[\frac{1}{2} F_{\mu \nu}^{2}+\left(D_{\mu} \Phi_{i}\right)^{2}\right]
$$

In this expression we omit fermions. The indices $\mu, \nu=0,1$ are worldvolume coordinates while $i=2,3, \cdots, 9$ is for transverse directions in the bulk. This action describes the low energy dynamics of parallel two D-strings. The fields are decomposed into components by Pauli matrices as $A_{\mu}=(1 / 2) \sigma^{a} A_{\mu}^{a}$ and a similar expression for $\Phi_{i}$, with the definition of the covariant derivative as

$$
F_{\mu \nu}^{a} \equiv \partial_{\mu} A_{\nu}^{a}-\partial_{\nu} A_{\mu}^{a}+\epsilon^{a b c} A_{\mu}^{b} A_{\nu}^{c}, \quad D_{\mu} \Phi^{a} \equiv \partial_{\mu} \Phi^{a}+\epsilon^{a b c} A_{\mu}^{b} \Phi^{c} .
$$

The locations of the D-strings $Y^{i}$ are specified by the eigenvalues of the Higgs field with the rescaling $2 \pi \alpha^{\prime} \Phi_{i}=Y^{i}$. In the following we turn on only $Y^{9}$ and omit the transverse index $i$.

\footnotetext{
${ }^{\ddagger}$ In this paper except Section 4, we omit writing tensions of D-branes which appear as an overall factor of the actions.
} 
The background representing the intersecting D-strings should be

$$
\Phi^{3}=q x, \quad A_{\mu}=0 .
$$

Here $x$ denotes $x^{1}$. It is obvious that this is a solution of the equations of motion for the action (2.2). This solution breaks all the supersymmetries. Taking into account the rescaling factor $2 \pi \alpha^{\prime}$, one finds that the constant slope $q$ is related to the intersection angle $\theta$ as

$$
q=\frac{1}{\pi \alpha^{\prime}} \tan (\theta / 2)
$$

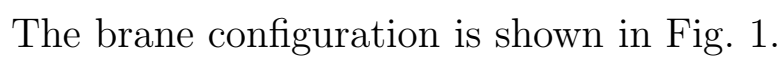

Let us proceed to the analysis of the fluctuations around this background (2.4). We turn on only $A_{1}^{2} \equiv a$ and $\Phi^{1} \equiv \varphi$ since other fluctuation fields decouple from these ${ }^{\S}$ at the quadratic level. The Lagrangian quadratic in the fluctuations is calculated as

$$
L=\frac{1}{2}\left(\partial_{0} a\right)^{2}+\frac{1}{2}\left(\partial_{0} \varphi\right)^{2}-\frac{1}{2}\left(\left(\partial_{x} \varphi\right)^{2}+2 q x a \partial_{x} \varphi+q^{2} x^{2} a^{2}-2 q a \varphi\right) .
$$

The equations of motion for the fluctuations are

$$
\left(\begin{array}{cc}
-q^{2} x^{2}-\partial_{0}^{2}-q x \partial_{x}+q \\
q x \partial_{x}+2 q & \partial_{x}^{2}-\partial_{0}^{2}
\end{array}\right)\left(\begin{array}{c}
a(t, x) \\
\varphi(t, x)
\end{array}\right)=0 .
$$

To obtain the mass spectrum, we decompose the fluctuation fields into the linear combination of mass eigen functions as

$$
\left(\begin{array}{l}
a(t, x) \\
\varphi(t, x)
\end{array}\right)=\sum_{n \geq 0}\left(\begin{array}{c}
\widetilde{a}_{n}(x) \\
\widetilde{\varphi}_{n}(x)
\end{array}\right) C_{n}(t)
$$

where the decomposed field $C_{n}(t)$ satisfies a free field equation

$$
\left(\partial_{0}^{2}+m_{n}^{2}\right) C_{n}(t)=0
$$

with the mass eigenvalues $m_{n}$ for each $n$. The eigen equations are given by

$$
\left(\begin{array}{cc}
-q^{2} x^{2}+m_{n}^{2}-q x \partial_{x}+q \\
q x \partial_{x}+2 q & \partial_{x}^{2}+m_{n}^{2}
\end{array}\right)\left(\begin{array}{c}
\widetilde{a}_{n}(x) \\
\widetilde{\varphi}_{n}(x)
\end{array}\right)=0 .
$$

Solving this matrix differential equation, the mass squared is obtained as

$$
m_{n}^{2}=(2 n-1) q
$$

\footnotetext{
$\S$ Another pair $\left(A_{1}^{1}, \Phi^{2}\right)$ which is decoupled from the above appears with a similar Lagrangian. This another pair provides doubly degenerated spectrum, which is consistent with the string spectrum that is also doubly degenerated due to the possible orientations of the string. We shall see that in each sector a tachyonic mode appears which is combined into a complex tachyon field.
} 
for normalizable eigen functions $\left(\widetilde{a}_{n}(x), \widetilde{\varphi}_{n}(x)\right)$, with $n=0,2,3,4,5, \cdots$. The explicit expression for the eigen functions are

$$
\begin{aligned}
& \widetilde{a}_{n}(x)=-e^{-q x^{2} / 2} \sum_{j=0,2, \cdots}^{n}(-1)^{\frac{j}{2}} \frac{4^{\frac{j}{2}}}{j !} \frac{n(n-2) \cdots(n-j+2)}{2 n-1}(j-1)(x \sqrt{q / 2})^{j}, \\
& \widetilde{\varphi}_{n}(x)=e^{-q x^{2} / 2} \sum_{j=0,2, \cdots}^{n}(-1)^{\frac{j}{2}} \frac{4^{\frac{j}{2}}}{j !} \frac{n(n-2) \cdots(n-j+2)}{2 n-1}(2 n-j-1)(x \sqrt{q / 2})^{j}
\end{aligned}
$$

for $n=0,2, \cdots$, and

$$
\begin{aligned}
& \widetilde{a}_{n}(x)=-e^{-q x^{2} / 2} \sum_{j=1,3, \cdots}^{n}(-1)^{\frac{(j-1)}{2}} \frac{4^{\frac{(j-1)}{2}}}{j !}\left(\frac{j-1}{2}\right)(n-3) \cdots(n-j+2)(x \sqrt{q / 2})^{j}, \\
& \widetilde{\varphi}_{n}(x)=e^{-q x^{2} / 2}\left(\sqrt{q / 2} x+\sum_{j=3,5, \cdots}^{n} \frac{(-4)^{\frac{(j-1)}{2}}}{j !}\left(n-\frac{j+1}{2}\right)(n-3) \cdots(n-j+2)(x \sqrt{q / 2})^{j}\right)
\end{aligned}
$$

for $n=3,5, \cdots$. Substituting the relation (2.5) into the mass formula (2.11), we find that it agrees with the string worldsheet spectrum (2.1) up to $\mathcal{O}\left(\theta^{3}\right)$ ambiguity. In order to match two results to higher order in $\theta$, we should have to adopt the nonAbelian Born-Infeld Lagrangian as a starting point. In appendix A, we include the first nontrivial $\alpha^{\prime}$ corrections of order $\left(\alpha^{\prime}\right)^{2} F^{4}$ from string theory, and showed that the fluctuation spectrum agrees with the worldsheet result up to $\mathcal{O}\left(\theta^{5}\right)$ ambiguity.

Our concern is the lowest mode $n=0$ which is tachyonic. Surprisingly, the eigen functions for this lowest mode is quite simple,

$$
\widetilde{\varphi}_{0}(x)=\widetilde{a}_{0}(x)=\exp \left[-\frac{q x^{2}}{2}\right]\left(=\exp \left[-\frac{\tan (\theta / 2)}{2 \pi \alpha^{\prime}} x^{2}\right]\right) .
$$

The fluctuation modes are localized at $x=0$ which is the intersection point, which agrees again with the worldsheet perspective. If $\theta$ is small enough, the above fluctuation mode does not invalidate the low energy approximation of the D-brane action as Yang-Mills theory, since the derivatives (involved with the string $\alpha^{\prime}$ corrections) acting on the fluctuation give only small contributions. In this respect, the approximation is invalid for $\theta$ exceeding $\pi / 2$.

The fact that the eigen functions are Gaussian is consistent with the T-dual results in Ref. [7] where the eigen functions are $\Theta$ functions. The $\Theta$ functions are roughly a summation of periodically placed Gaussian functions, and Ref. [7] considered D-branes wrapping a torus for the T-duality so the above localized eigenfunctions should appear periodically there.

\subsection{Realization of D-string recombination}

Let us find the constraint on the magnitude $C \equiv C_{0}(t)$ of the tachyonic fluctuation (2.12) first. This is derived by requiring that the quadratic approximation is valid, 


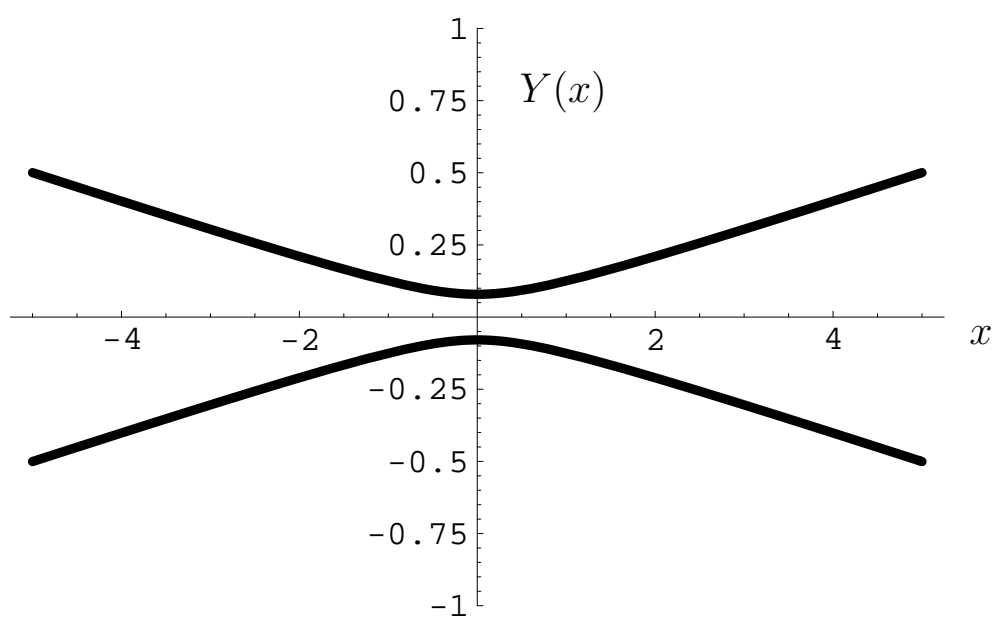

Figure 2: Intersecting D-strings are recombined. Here we draw the configuration (2.18) with parameters $q=\tan (\theta / 2)=0.1$ and $C=\sqrt{q} / 4$ (with $\pi \alpha^{\prime}=1$ ).

that is, the terms quartic in fluctuations are much smaller than quadratic terms. Straightforward calculation shows that in the original Yang-Mills Lagrangian the quadratic terms with eigen functions (2.12) are

$$
\text { (quadratic terms) } \sim q C^{2} e^{-q x^{2}},
$$

while the quartic terms are

$$
\text { (quartic terms) } \sim C^{4} e^{-2 q x^{2}} .
$$

Hence the above consistency requires

$$
q \gg C^{2} .
$$

This is the condition for that the approximation we adopted for the fluctuation analysis is valid. The free field equation (2.9) for $C(t)$ is solved with an exponentially growing function

$$
C \sim e^{\sqrt{q} t},
$$

and our approximation is valid until this $C(t)$ reaches the critical value $\sqrt{q}$ as seen in Eq. (2.15).

Keeping this condition in mind, let us study the geometric picture of the condensation of the tachyonic fluctuation. The locations of the D-strings are specified by the eigenvalues of the Higgs field $\varphi$. We diagonalize the whole Higgs field including the fluctuation (2.12), as

$$
\Phi(t, x)=\frac{1}{2}\left(\begin{array}{cc}
q x & \varphi_{0}(x) \\
\varphi_{0}(x) & -q x
\end{array}\right) \rightarrow \frac{1}{2}\left(\begin{array}{cc}
\sqrt{q^{2} x^{2}+C^{2} e^{-q x^{2}}} & 0 \\
0 & -\sqrt{q^{2} x^{2}+C^{2} e^{-q x^{2}}}
\end{array}\right)
$$

\footnotetext{
IThe gauge fields are not diagonalized simultaneously.
} 


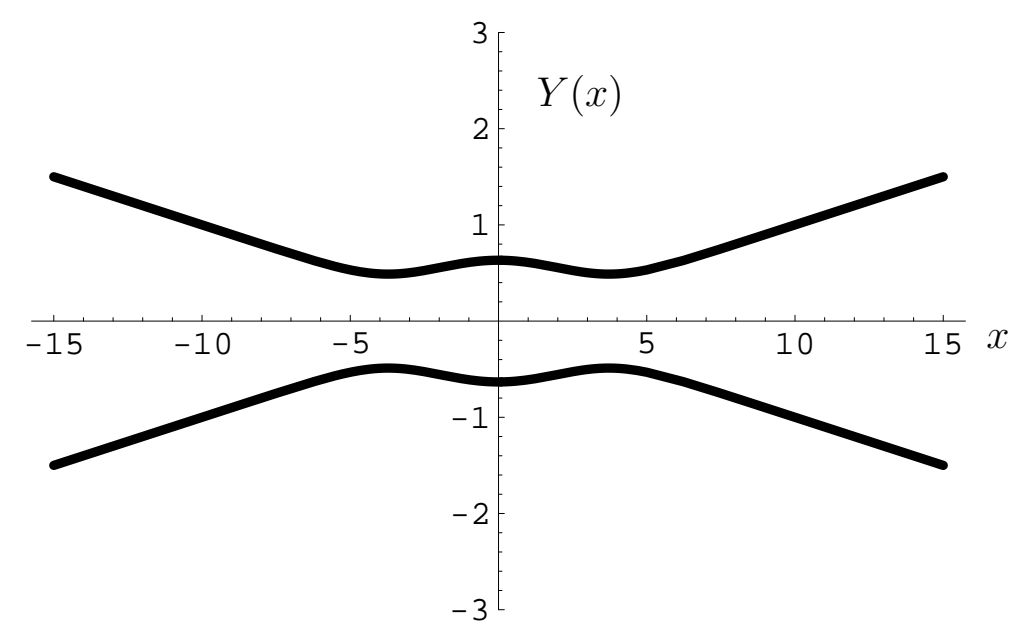

Figure 3: For large $C$, physically unacceptable recombination is provided by (2.18). Here we have chosen the same value of $q$ as in the previous figure but with $C=2 \sqrt{q}>\sqrt{q}$. This exceeds the validity of the approximation and thus prohibited. Before the configuration reaches this form, the nonlinear effect of fluctuations becomes important.

Therefore after the tachyon condensation, two D-strings are located at

$$
Y(x)= \pm \pi \alpha^{\prime} \sqrt{q^{2} x^{2}+C^{2} e^{-q x^{2}}}= \pm \sqrt{(\tan (\theta / 2) x)^{2}+C^{2} \exp \left[-\frac{\tan (\theta / 2)}{\pi \alpha^{\prime}} x^{2}\right]}
$$

This configuration of D-strings is shown in Fig. 2. The intersecting D-strings are recombined! Naively it might have been expected that the recombination might be difficult to be realized in Yang-Mills theory since the locations of the D-strings are represented by the eigen values and the recombination process must interchange two eigenvalues only in a half of the worldvolume $(x<0)$. However this is possible, as seen in the above diagonalization, due to the gauge transformation which was singular at $x=0$ originally before the tachyon condensation (the limit $C \rightarrow 0$ ). So essentially the tachyon condensation resolves the singularity of the gauge transformation and enables the interchange of the left "halves" of the D-strings (the $x<0$ region).

The intriguing point here is that the magnitude of the tachyon mode $C$ corresponds just to the interbrane separation. This can be easily seen in Eq. (2.18) with $x=0$. Therefore, in the D-brane recombination, the tachyon condensation is geometrically realized.

It is interesting to note that the condition (2.15) has the following geometrical meaning. From the expression (2.18) of the transverse displacement, we observe that when the tachyon develops the condensation and $C$ becomes large enough, the Dstring worldvolume embedding is "waving" in the bulk (See Fig. 3). This situation is quite strange in the naive view of the energy minimization in which the D-string 
energy would be given mostly as a tension times its length. The "wave" should be smeared. The requirement that this "wave" does not appear is equivalent to the condition that the function $Y(x)(2.18)$ has only a single extremum. This condition is easily evaluated as $q>C^{2}$ which is satisfied by (2.15).

\section{Large intersection angle and Sen's conjecture}

In the previous section we showed that the recombination of the intersecting D-strings is realized in Yang-Mills theory, the low energy scheme of string theory. However as mentioned in the introduction, this phenomenon of recombination should be related to the tachyon condensation of brane-antibrane system. The worldsheet picture (2.1) tells us that when $\theta=\pi$, the tachyon mass squared reaches its lower bound $m^{2}=$ $-1 / 2 \alpha^{\prime}$ which is well-known as that of tachyons whose condensation is conjectured to make the brane-antibrane disappear (the Sen's conjecture, see Ref. [6]).

Then, how the disappearance of the brane-antibrane is related to the recombination? The answer is found when we consider D-strings intersecting at large $\theta, \theta \sim \pi$. For this large $\theta$, the worldvolume parametrization with $x$ (the horizontal axis) is not appropriate. Instead, we exchange the roles of $x$ and $Y(x)$ : now let us parametrize the worldvolume of D-strings by $y$, the vertical axis.* Then the displacement of the D-strings from this axis is measured by a scalar field $X(y)$ which was originally $x$ (See the left of Fig. (4). One notices that in this parametrization, two D-strings become a pair of a D-string and an anti-D-string, since the orientation of one D-string is reversed compared to the other, as is obvious in Fig. 4 .
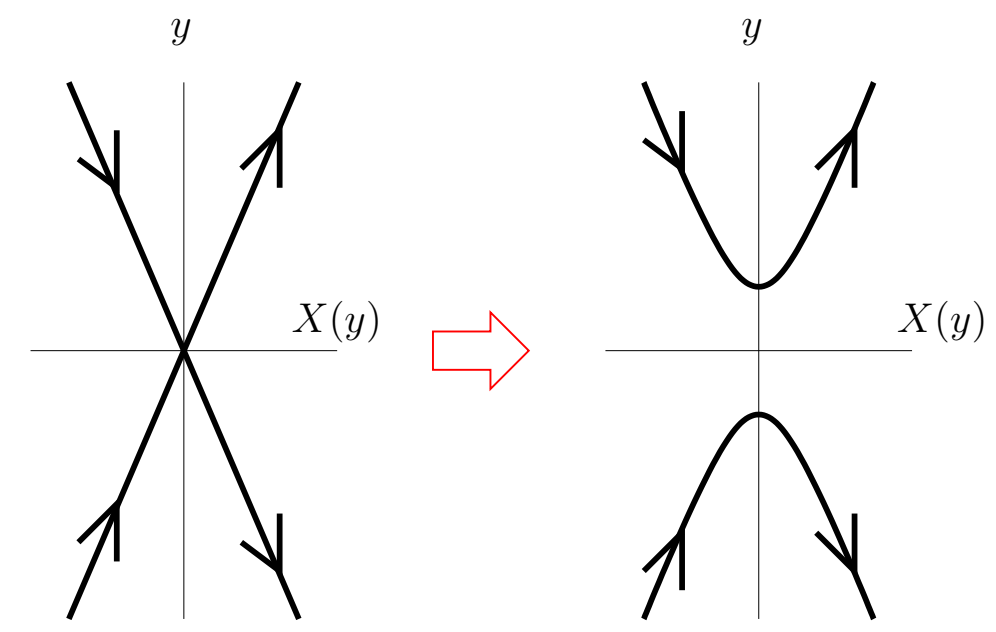

Figure 4: D-string recombination with $\theta \sim \pi$. By rotating these figures by $\pi / 2$, one can see the brane-antibrane annihilation occurring around the origin.

*This kind of the change of the axes of the worldvolume parametrization has been investigated in Ref. 12] as "target space rotation." 
The tachyonic mode $n=0$ of (2.1) remains at the origin and tends to be condensed. Since we have explicitly shown in the previous section that the condensation of this mode is equivalent to the D-string recombination, so assuming that even with large $\theta$ this equivalence is maintained, the condensation of this tachyonic mode for large $\theta$ should provide again the recombination depicted in the right of Fig. 1 . Reminding of the fact that the natural parametrization of the worldvolume is $y$, we observe from this figure that around $y=0$ where the tachyon is localized the braneantibrane has disappeared! We regard this as a qualitative verification of the Sen's conjecture on the tachyon condensation.

The single assumption which we made in the argument above was that the identification of the fluctuation mode (2.12) with the lowest mode of the worldsheet spectrum (2.1) is valid also for large $\theta$. Although it is hard to imagine that this is violated, in the following we provide another evidence that the identification for large $\theta$ is valid.

For larger $\theta$, the approximation adopted in the previous section is getting worse, and so we cannot use the Yang-Mills approach. Hence we necessarily have to use alternative picture mentioned above, the worldvolume parametrization in terms of $y$. This brane-antibrane picture is difficult to be analysed since it needs stringy description with the energy scale $\alpha^{\prime}$, such as string field theories. However, we shall see that a very simple tachyon action derived from string scattering amplitudes turns out to be useful for our purpose. The tachyon two point function on a $\mathrm{D} p$-antiD $p$ pair is reproduced by the free theory

$$
S=-\int d t d^{p} y\left[\frac{1}{2}\left|\partial_{\mu} T\right|^{2}-\frac{1}{4 \alpha^{\prime}}|T|^{2}\right]
$$

Here the tachyon field is complex since the strings joining the D-strings are orientable. This tachyon is charged under a combination of the gauge fields, $A \equiv A^{(1)}-A^{(2)}$ where $A_{\mu}^{(i)}$ is the $U(1)$ gauge field on the $i$-th $\mathrm{D} p$-brane $(i=1,2)$. Taking this into account, the above action is generalized to be gauge-invariant,

$$
S=-\int d t d^{p} y\left[\frac{1}{4} F_{\mu \nu}^{2}+\frac{1}{2}\left|D_{\mu} T\right|^{2}-\frac{1}{4 \alpha^{\prime}}|T|^{2}\right],
$$

where the covariant derivative is defined as

$$
D_{\mu} T \equiv \partial_{\mu} T-i A_{\mu} T
$$

For describing the nontrivial transverse displacement $X(y)$ from the $y$ axis, we make a T-duality (dimensional reduction) in which $X(y)$ is identified as a gauge field, $2 X(y)=2 \pi \alpha^{\prime} A_{1}$. The resultant action is

$$
S=-\int d t d y\left[\frac{1}{4} F_{\mu \nu}^{2}+\frac{2}{\left(2 \pi \alpha^{\prime}\right)^{2}}\left(\partial_{\mu} X\right)^{2}+\frac{1}{2}\left|D_{\mu} T\right|^{2}-\frac{1}{4 \alpha^{\prime}}|T|^{2}+\frac{2}{\left(2 \pi \alpha^{\prime}\right)^{2}} X^{2}|T|^{2}\right](3
$$


This action is defined on a $1+1$ dimensional worldvolume spanned by $y$ and $t$. The field $X(y)$ describes the distance between the (anti-)D-string and the $y$ axis. A solution which represents the intersecting D-strings is obtained as

$$
X(y)=\tan (\phi / 2) y, \quad T=0, \quad F_{a b}=0,
$$

where we have defined $\phi=\pi-\theta$ which is small.

This solution (3.5) is expected to modify the tachyon mass squared and give the localization of the tachyon around $y=0$. Let us check these. The real part of the tachyon fluctuation $T$ around the solution (3.5) is decoupled from other fluctuations, and the Lagrangian for this fluctuation is given $b^{\dagger}$

$$
S_{\text {fluc }}=-\int d^{2} x\left[\frac{1}{2}\left(\partial_{\mu} T\right)^{2}-\frac{1}{4 \alpha^{\prime}} T^{2}+\frac{2 \tan ^{2}(\phi / 2) y^{2}}{\left(2 \pi \alpha^{\prime}\right)^{2}} T^{2}\right] .
$$

The eigenvalue problem of this harmonic potential is easily solved to give the lowest mass squared which is negative,

$$
m^{2}=-\frac{1}{2 \alpha^{\prime}}+\frac{1}{\pi \alpha^{\prime}} \tan (\phi / 2)=-\frac{\theta}{2 \pi \alpha^{\prime}}+\mathcal{O}\left((\pi-\theta)^{2}\right) .
$$

This agrees with the lowest tachyonic mode in the string spectrum (2.1).$^{\ddagger}$

The eigen function for this tachyonic mode (3.7) is found as

$$
T=\widetilde{C}(t) \exp \left[-\frac{\tan (\phi / 2)}{2 \pi \alpha^{\prime}} y^{2}\right]
$$

which is localized at $y=0$. Interestingly, this wave function of the tachyonic mode is precisely the same as that of the lowest fluctuation of the Yang-Mills analysis (2.12). To show this, first we have to note that the asymptotic configuration of the D-strings is given in both cases (2.18) and (3.5) by

$$
y= \pm \tan (\theta / 2) x
$$

where we have used the definition $\phi \equiv \pi-\theta$ which gives

$$
\tan (\theta / 2)=[\tan (\phi / 2)]^{-1}
$$

This strongly suggests that the wave function of the tachyonic fluctuation mode obtained in Yang-Mills scheme (2.12) is not deformed even for large $\theta$. In addition, the tachyon condensation parameter $\widetilde{C}$ is expected to be proportional to $C$.

\footnotetext{
${ }^{\dagger}$ Since the solution is $T=0$, we denote the tachyon fluctuation also as $T$.

$\ddagger$ The fluctuation analysis gives an equally-spaced spectrum $m^{2}=-\frac{1}{2 \alpha^{\prime}}+\frac{2 k+1}{\pi \alpha^{\prime}} \tan (\phi / 2)$ with a non-negative integer $k$. Note that this $k$ does not coincide with $n$ in Eq. (2.1). Only the lowest mode $k=0$ corresponds to the lowest mode $n=0$.
} 
As we mentioned, other fluctuations such as the scalar fluctuation $\delta X(y) \equiv$ $X(y)-2 \tan (\phi / 2) y$ is decoupled from the tachyon fluctuation. But once the tachyonic fluctuation is condensed as (3.8) with nonzero $\widetilde{C}$, this induces nontrivial effect on $\delta X$. One can solve this backreaction to $X(y)$ provided the tachyon condensation (3.8). This gives some information on the brane disappearance around $y=0$. The result is

$$
\delta X(y) \sim \frac{\pi \alpha^{\prime}}{2} \widetilde{C}^{2} \int^{y} d \hat{y} \exp \left[-\frac{\tan (\phi / 2)}{\pi \alpha^{\prime}} \hat{y}^{2}\right] .
$$

Interestingly, this is an error function and thus the region where $\delta X$ is non-vanishing is not localized. Since the localized tachyon condensation should not give nonlocal translation of the D-strings, the above result indicates that the worldvolume gets discontinuous around $y=0$ : the result (3.11) should be split into two regions so that the effect is localized,

$$
\delta X(y) \sim\left\{\begin{array}{c}
-\frac{\pi \alpha^{\prime}}{2} \widetilde{C}^{2} \int_{-\infty}^{y} d \hat{y} \exp \left[-\frac{\tan (\phi / 2)}{\pi \alpha^{\prime}} \hat{y}^{2}\right](y<0) \\
\frac{\pi \alpha^{\prime}}{2} \widetilde{C}^{2} \int_{y}^{\infty} d \hat{y} \exp \left[-\frac{\tan (\phi / 2)}{\pi \alpha^{\prime}} \hat{y}^{2}\right](y>0)
\end{array} .\right.
$$

We have added different constant parameters for the two regions so that asymptotically $(y \rightarrow \pm \infty) \delta X(y)$ vanishes. Noting the following rough approximation for large $z$

$$
\int_{z}^{\infty} d \hat{z} e^{-c \hat{z}^{2}} \sim \frac{1}{2 c z} e^{-c z^{2}}
$$

$\delta X(y)$ in Eq. (3.12) is approximated for large $y$ as

$$
\delta X(y) \sim\left\{\begin{array}{c}
-\frac{\left(\pi \alpha^{\prime}\right)^{2}}{4 \tan (\phi / 2)} \widetilde{C}^{2} \cdot \frac{1}{y} \exp \left[-\frac{\tan (\phi / 2)}{\pi \alpha^{\prime}} y^{2}\right](y<0) \\
\frac{\left(\pi \alpha^{\prime}\right)^{2}}{4 \tan (\phi / 2)} \widetilde{C}^{2} \cdot \frac{1}{y} \exp \left[-\frac{\tan (\phi / 2)}{\pi \alpha^{\prime}} y^{2}\right](y>0)
\end{array} .\right.
$$

Surprisingly, this form coincides with the Yang-Mills result (2.18) for large $x$ as seen below. We expand (2.18) for large $x$ as

$$
\frac{Y(x)}{\tan (\theta / 2)}= \pm\left(x+\frac{C^{2}}{2 \tan ^{2}(\theta / 2)} \frac{1}{x} \exp \left[-\frac{\tan (\theta / 2)}{\pi \alpha^{\prime}} x^{2}\right]+\text { higher }\right) \text {. }
$$

Rewriting this with the identification used previously, (3.9) and (3.10), one can show that the second term in Eq. (3.15) is identical with (3.14) with a certain linear relation between the two constant parameters $C$ and $\widetilde{C}$. The second term in Eq. (3.15) is of order $C^{2}$ due to the diagonalization (2.18). To compare this with the tachyon picture, we had to consider the backreaction which is higher order in $\widetilde{C}$. 
With the simple tachyon action (3.4) employed, we have shown various coincidence with the fluctuation analysis of the previous section. It would be interesting to study this tachyon system more carefully, for example with use of the boundary string field theory for brane-antibranes. A trial along this direction is presented in Appendix B.

\section{Dynamical aspects of recombination: decay width}

We have identified the tachyonic modes which triggers the D-brane recombination and showed its significance in relation to the Sen's conjectures. In application to D-brane cosmology for example, it is important to study how the time-dependent recombination process proceeds. This question is related to the recent development on rolling tachyons [13], however our privilege is that we can use low energy description instead of fully following the worldsheet string theory. The recombination process itself is just the vacuum condensation of the tachyonic mode (2.12), however once the tachyon expectation value $C(t)$ reaches its critical value $\sqrt{q}$, the nonlinear effect (or equivalently, higher order terms of fluctuations) becomes important. After a while, the off-diagonal modes of the stretched open string will decouple and the system will be dictated by a Nambu-Goto action for a D-string. The relative velocity between D-strings leaving each other will soon reach the speed of light (in our approximation of decoupling limit of closed strings), as discussed in Ref. [14]. This is a geometrical realization of rolling tachyon [13], since in the present case the magnitude of the tachyon mode is identical with the interbrane separation, as seen in Section 2.

In the argument above we started from intersecting D-strings, however this configuration is of course unstable and it is another question whether this is a reasonable initial condition to be started with or not. In the context of string cosmology, it is expected that first many D-branes are created in a hot universe and then they interact with each other by recombination. Therefore more physical initial condition might be non-parallelly separated D-branes.

In this section we present a naive estimation of the decay width of the nonparallelly separated D-branes. In fact the following process might be physically plausible: two D-strings which are not parallel approach each other by gravitational and Ramond-Ramond forces, and just at the time when they intersect, the tachyon field at the intersection point starts rolling down the potential hill and condenses to provide the recombination. However, as discussed in Ref. [14, 15], before they intersect there should be a small probability for quantum tunnelling directly into the recombined final state. For analysing this process, we place two D-strings apart from each other so that the open string connecting the two does not yet have any tachyonic excitation. We fix the distance between the branes as in Fig. 5 for simplicity. In this situation, the branes cannot approach each other and thus the decay mode is due to the quantum tunnelling effect. We generalize the computation in Ref. [14] to 


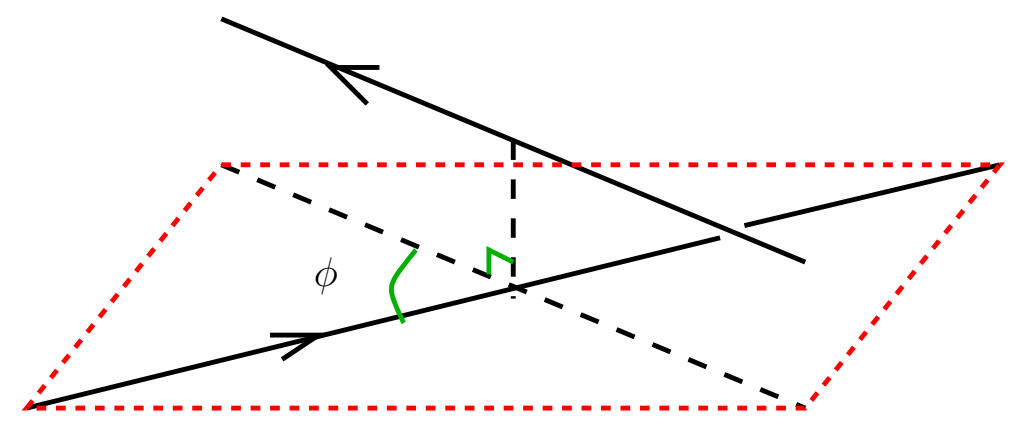

Figure 5: Intersecting branes are separated.

the case of the branes not only separated anti-parallelly but also tilted against each other. The intersection angle $\phi$ will appear in the decay width.

The quantum tunnelling is dictated by a bounce solution whose geometric interpretation is the Euclidean worldvolume deformation of the D-string, that is, a formation of a throat connecting the two D-strings. When $\phi=0$, the D-strings are placed anti-parallelly, and the decay width can be evaluated in the following way: First, the decay width is the exponential of the on-shell Euclidean action,

$$
\Gamma=\exp \left(-S_{\mathrm{E}}\right)
$$

where $S_{\mathrm{E}}$ is the action into which the throat (volcano) solution of the worldvolume field theory is substituted (see Ref. [16] for this type of solutions). The throat action is evaluated easily as

$$
S_{\mathrm{E}}=T_{\mathrm{D} 1}\left(2 \pi R d-2 \pi R^{2}\right),
$$

where $d$ is the distance between the branes, and $R$ is the radius of the throat. The first term is from the area of the cylinder (throat), and the second term is from the areas of the cap and the bottom of the throat which are holes and thus give a negative contribution. The throat radius $R$ can be determined by extremization of the action $S_{\mathrm{E}}$ as

$$
\frac{\partial S_{\mathrm{E}}}{\partial R}=0
$$

which gives $R=d / 2$. Then the on-shell action is

$$
S_{\mathrm{E}}=T_{\mathrm{D} 1} \frac{\pi d^{2}}{2}
$$

Thus a trivial result is that when the distance between the two becomes large, the decay width by a formation of a throat is exponentially dumping, as $\Gamma \sim$ $\exp \left(-T_{\mathrm{D} 1} d^{p+1}\right)$. 
Now let us introduce the intersection angle to the system. It is expected that the location of the throat formed by the tunnel effect is unique, at the intersection point. One way to explain this is that, from the geometrical point of view, it is unlikely for the throat to be formed far from the intersection point since it needs extra energy to create it (in other words, the potential barrier is higher). Another way to explain this is that the string connecting the two is localized around the intersection point, as seen from both the Yang-Mills picture and the tachyon picture studied in the previous sections.

So here we simply assume that the throat is formed at the intersection point (though they are not actually intersecting but separated by $d$ ). Further assumption is that the cylinder is squeezed and become elliptic due to the localization of the string. We assume that the horizontal section of this cylinder which was a circle for $\phi=0$ is deformed to be an ellipse,

$$
\tau^{2}+\frac{y^{2}}{f^{2}}=R^{2}
$$

Here $\tau$ is the Euclidean time, and $f$ is a function of $\phi$ which will be determined later by the extremization process.

The area of two holes can be easily evaluated as

$$
S_{\mathrm{E}}^{(\text {hole })}=-T_{\mathrm{D} 1} 2 \pi R^{2} f
$$

where the last factor $f$ comes from the elliptic deformation. On the other hand, the area of the side of the cylinder is

$$
S_{\mathrm{E}}^{(\text {side })}=T_{\mathrm{D} 1} \cdot 4 \int_{0}^{R} d \tau \sqrt{1+\left(\frac{d y}{d \tau}\right)^{2}} \sqrt{d^{2}+4 \tau^{2} \tan ^{2}(\phi / 2)}
$$

where $y$ is now defined by $\tau$ through (4.5). The equations of motion for $f$ and $R$ are given by

$$
\frac{\partial}{\partial R} S_{\mathrm{E}}=0, \quad \frac{\partial}{\partial f} S_{\mathrm{E}}=0
$$

where $S_{\mathrm{E}}=S_{\mathrm{E}}^{(\text {side })}+S_{\mathrm{E}}^{(\text {hole })}$. These are integral equations, and the integrands are difficult to be exactly evaluated. So let us concentrate on only small $\phi$. It is natural to expect a correction

$$
f=1+\mathcal{O}\left(\phi^{2}\right)>1
$$

which means that the cylinder is squeezed, in other words, enlarged along the direction $y$. So we define $1+2 g \equiv f^{2}$ and expand the integrand so that the integration is 
possible. The result for the first equation in $(4.8)$ is

$$
\begin{aligned}
2 \pi d[ & +\frac{1}{2}\left(g+6 \frac{R^{2}}{d^{2}} \tan ^{2}(\phi / 2)\right) \\
& \left.+\frac{3}{8}\left(-\frac{1}{2} g^{2}+6 g \frac{R^{2}}{d^{2}} \tan ^{2}(\phi / 2)-10 \frac{R^{4}}{d^{4}} \tan ^{4}(\phi / 2)\right)-\frac{2 R}{d}\left(1+g-\frac{1}{2} g^{2}\right)\right]=0 .
\end{aligned}
$$

The second equation in (4.8) gives

$$
\frac{\pi}{8} d R\left[8+2 g+12 \frac{R^{2}}{d^{2}} \tan ^{2}(\phi / 2)-\frac{5}{2} g+2 g \frac{R^{2}}{d^{2}} \tan ^{2}(\phi / 2)-10 \frac{R^{4}}{d^{4}} \tan ^{4}(\phi / 2)-16 \frac{R}{d}\right]=0 \text {. }
$$

In both equations we neglect $\mathcal{O}\left(\phi^{6}\right)$ terms. Then these two can be solved as

$$
\begin{aligned}
& R=\frac{d}{2}\left(1+\frac{1}{2} \tan ^{2}(\phi / 2)+\frac{3}{8} \tan ^{4}(\phi / 2)+\mathcal{O}\left(\phi^{6}\right)\right), \\
& g=\frac{1}{2} \tan ^{2}(\phi / 2)+\frac{1}{2} \tan ^{4}(\phi / 2)+\mathcal{O}\left(\phi^{6}\right) .
\end{aligned}
$$

Hence the cylinder is squeezed as expected $(g>0)$,

$$
f=1+\frac{1}{2} \tan ^{2}(\phi / 2)+\frac{3}{8} \tan ^{4}(\phi / 2)+\mathcal{O}\left(\phi^{6}\right) .
$$

The final on-shell action into which these values are substituted is

$$
S_{\mathrm{E}}=T_{\mathrm{D} 1} \frac{\pi d^{2}}{2}\left(1+\frac{1}{2} \tan ^{2}(\phi / 2)+\frac{3}{8} \tan ^{4}(\phi / 2)+\mathcal{O}\left(\phi^{6}\right)\right) .
$$

This result shows that a larger $\phi$ gives a smaller probability of the throat formation. ${ }^{\S}$ This result is consistent with the observation that, a system closer to a supersymmetric configuration of parallel parallel two D-strings $(\phi=\pi)$ is more stable so the most unstable system should be the parallel D1-antiD1 configuration $(\phi=0)$. As $\phi$ increases, the tachyonic instability of the system decreases and thus the throat nucleation probability should decrease accordingly.

We note here that we cannot compare the above result with the case with exactly vanishing $\phi$, since $\phi=0$ case gives a probability per a unit world volume of D-strings while in our present case the formation occurs only at the intersection point. So the physical meaning of the resultant decay width is different from the $\phi=0$ case.

\footnotetext{
${ }^{\S}$ It is interesting to note that all the above results $(4.10),(4.12)$ and $(4.13)$ has the same corrections in $\phi$. This indicates some deeper physical reason. We may expect that the corrections can be summarized universally as

$$
\left(1+\frac{1}{2} \tan ^{2}(\phi / 2)+\frac{3}{8} \tan ^{4}(\phi / 2)+\mathcal{O}\left(\phi^{6}\right)\right)=F(\phi)
$$

with $\lim _{\phi \rightarrow \pi} F(\phi)=+\infty$, so that the system approaches BPS parallel D-branes giving a vanishing decay width.
} 
The width we have found here is very small, since we are working in the decoupling limit $g_{\text {string }} \rightarrow 0$. This is potentially due to the fact that the distance between the D-strings is fixed. One of the other possibilities of the initial conditions is to consider D-strings approaching each other with a constant relative velocity $v$. If this $v$ is small enough, there should be enough long period for the D-strings to be recombined by developing the tachyon condensation. The period during which the tachyonic mode is present is evaluated as $\sim \theta l_{\text {string }} / v$. The condensation should start by a quantum fluctuation studied in Ref. [17], whose characteristic time is roughly $\sim l_{\text {string }} / \theta$, hence we expect that the D-strings recombination occurs when $\theta^{2}>v$.

\section{Future directions}

The mechanism of the recombination of the intersecting D-branes provided in this paper is quite simple, basically due to the fact that we can employ low energy approximation of the D-brane dynamics which is the super Yang-Mills theory. Therefore we expect that it is possible to apply the recombination mechanism presented in this paper in many directions which include (i) study for formulating string/M-theory in terms of higher dimensional D-branes as fundamental objects, (ii) verification of the Sen's conjectures using various schemes, and (iii) string phenomenology based on intersecting branes, and brane cosmology.

As for (i), the first step might be to generalize the present analysis to higher dimensional D-branes. " For example, D2-branes intersecting in $T^{4}$ can be supersymmetric with a certain relation of two intersection angles [8]. It is interesting to see this phenomena in terms of low energy Yang-Mills theory.

Regarding (ii), since there has been vast amount of literature concerning the Sen's conjectures, the relation between those and our mechanism should be explored further. We may turn on the intersection angle for example in the scheme of cubic string field theories or boundary string field theories, which will help to clarify the relation. On the other hand, our analysis shows that the tachyon condensation of brane-antibrane can be seen by the Yang-Mills theories, which suggests that they can describe various phenomena associated with the tachyon condensation, such as the brane descent relations and creation of lower-dimensional D-branes. Furthermore, it might be possible even to describe all the tachyon condensation processes by (generalizations of) Yang-Mills theories. For example, non-BPS branes defined as unstable defects in tachyon condensation of brane-antibrane can also be described in our Yang-Mills scheme, and this might be the case also for the rolling tachyons [13] and associated S(pacelike)-branes [19] whose deformations describe creation of lower dimensional defects [20]. In our realization, the rolling tachyon is realized geometrically as recombined D-branes leaving each other. The rolling tachyon in the present

IInstabilities in the higher dimensional cases were discussed in Ref. [18] in the context of Mtheory lift. 
case should be described by a Dirac-Born-Infeld action in which the scalar field is identified with the tachyon, as seen in Section 2. This might give a path to justify the tachyon action of Ref. [13].

Finally, there should be much possibilities for application to phenomenology and cosmology (iii) since nowadays intersecting branes are considered to be an effective way of realizing our world on branes. Recombination of topological defects has been largely studied especially in the context of cosmic string network formation in cosmology [21]. In string theory, BPS D-branes can be realized as topological solitons in unstable D-branes with tachyons, based on the Sen's conjectures [6] on tachyon condensation. This viewpoint realizes the formation and time evolution of D-brane networks in the early universe, and the clarification of the recombination mechanism in this paper will help analysing phenomenological parameters. We hope that our research may be the first step to mediate between the string phenomenology and the tachyon condensation in string theory.

\section{Acknowledgments}

We would like to thank Washington Taylor IV for quite valuable discussions which were indispensable for completion of the present work, and appreciate useful comments by Akikazu Hashimoto, Tatsuya Noguchi and Gary Shiu. K. H. is supported in part by the Grant-in-Aid for Scientific Research (No. 12440060 and 13135205) from the Japan Ministry of Education, Science and Culture. After this paper was published, we were informed of the paper [24] in which instability of intersecting branes was studied in Yang-Mills theory.

\section{A. Higher order in $\theta$ and NBI corrections}

Here we discuss how the higher order terms in $\theta$ in the string theory spectrum (2.1) can be reproduced from the non-Abelian Born-Infeld action. The first nontrivial corrections to the Yang-Mills theory appear as $F^{4}$ terms in a symmetrized trace [9],

$$
L=\operatorname{Str} \sqrt{-\operatorname{det}\left(\eta_{\mu \nu}+2 \pi \alpha^{\prime} F_{\mu \nu}\right)} .
$$

The Lagrangian with the transverse scalar fields can be obtained simply by $\mathrm{T}$ dualities (dimensional reductions). To compute the higher order corrections, it is

of great help to use the expansion of Ref. [7] which provided a general expression of the fluctuation Lagrangian expanded around a diagonal and constant field strength background in $U(2)$ case. Here we can make use of it. The final form of the fluctuation Lagrangian in Ref. [7] is

$$
L=\operatorname{Str} \sqrt{-\operatorname{det}\left(\eta_{\mu \nu}+2 \pi \alpha^{\prime}\left(F_{0}\right)_{\mu \nu}\right)}\left[\frac{1}{2} B^{\mu \nu} \tilde{F}_{\nu \mu}-\frac{1}{4} g^{\mu \nu} \tilde{F}_{\nu \lambda} g^{\lambda \sigma} \tilde{F}_{\sigma \mu}\right]
$$


where $F_{0}$ is the background value of the field strength, $g$ is the open string metric, and $B^{\mu \nu} \equiv-g^{\mu \lambda} F_{\lambda \rho} \eta^{\rho \nu}$. In our case, the worldvolume is $1+2$ dimension before taking the T-duality down to $1+1$ dimensions, so we turn on only the field strength $\left(F_{0}\right)^{12}$. Then the open string metric reads

$$
g^{\mu \nu}=\operatorname{diag}(-1, g, g), \quad g \equiv \frac{1}{1+\left(2 \pi \alpha^{\prime}\left(F_{0}\right)_{12}\right)^{2}} .
$$

Then the fluctuation Lagrangian is written as

$$
\begin{aligned}
L=\operatorname{Str} & \frac{1}{\sqrt{g}}\left(g \cdot\left(F_{0}\right)_{12} i\left[A_{1}, \varphi\right]\right) \\
& \left.-\frac{1}{2} g\left(\partial_{0} A_{1}^{a}\right)^{2}-\frac{1}{2} g\left(\partial_{0} \varphi^{a}\right)^{2}-\frac{1}{2} g^{2}\left(\partial_{1} \varphi^{a}-i\left[A_{1}, \varphi\right]^{a}\right)^{2}\right) .
\end{aligned}
$$

We note here that the first line in the above expression receives no combinatoric factor when the symmetrized trace is taken, but the second line receives it. The prescription is given in Ref. [7]. When one has a symmetrized trace over a function of the form $\left(F_{0}\right)^{m} \tilde{F}\left(F_{0}\right)^{n} \tilde{F}$, then the $F_{0}$ dependence should appear as

$$
f\left(F_{0}\right) \rightarrow \frac{1}{F_{0}} \int_{0}^{F_{0}} d x f(x) .
$$

Following this prescription, we have

$$
\begin{aligned}
\sqrt{g} & \rightarrow 1-\frac{1}{6}\left(2 \pi \alpha^{\prime}\left(F_{0}\right)_{12}\right)^{2}-\frac{3}{40}\left(2 \pi \alpha^{\prime}\left(F_{0}\right)_{12}\right)^{4}+\mathcal{O}\left(\left(2 \pi \alpha^{\prime}\left(F_{0}\right)_{12}\right)^{6}\right) \\
g^{3 / 2} & \rightarrow 1-\frac{1}{2}\left(2 \pi \alpha^{\prime}\left(F_{0}\right)_{12}\right)^{2}-\frac{3}{40}\left(2 \pi \alpha^{\prime}\left(F_{0}\right)_{12}\right)^{4}+\mathcal{O}\left(\left(2 \pi \alpha^{\prime}\left(F_{0}\right)_{12}\right)^{6}\right)
\end{aligned}
$$

while the first line in Eq. (A.4) gives an expression as it is,

$$
\sqrt{g} F_{0}=F_{0}\left[1-\frac{1}{2}\left(2 \pi \alpha^{\prime}\left(F_{0}\right)_{12}\right)^{2}-\frac{3}{8}\left(2 \pi \alpha^{\prime}\left(F_{0}\right)_{12}\right)^{4}+\mathcal{O}\left(\left(2 \pi \alpha^{\prime}\left(F_{0}\right)_{12}\right)^{6}\right)\right] .
$$

Finally we obtain the fluctuation Lagrangian as (in the following we write only the terms up to the order $F^{4}$ explicitly, since it is known that the symmetrized trace prescription for corrections is valid only at this order [0])

$$
\begin{aligned}
L= & \left(1-\frac{1}{6}\left(2 \pi \alpha^{\prime}\left(F_{0}\right)_{12}\right)^{2}\right)\left[-\frac{1}{2}\left(\partial_{0} A\right)^{2}-\frac{1}{2}\left(\partial_{0} \varphi\right)^{2}\right. \\
& \left.\quad-\frac{1}{2}\left(1-\frac{1}{3}\left(2 \pi \alpha^{\prime}\left(F_{0}\right)_{12}\right)^{2}\right)\left(\left(\partial_{1} \varphi\right)^{2}+2 q x A \partial_{1} \varphi+q^{2} x^{2} A^{2}-2 q A \varphi\right)\right] \\
& +\mathcal{O}\left(\left(2 \pi \alpha^{\prime}\left(F_{0}\right)_{12}\right)^{4}\right) .
\end{aligned}
$$

So, up to the overall factor which is irrelevant for the fluctuation analysis, the difference between the present corrected Lagrangian and (2.6) is only that the mass 
squared will be multiplied by a factor appearing in the second line of Eq. (A.9). Noting that the correspondence here is that $\left(F_{0}\right)_{12}=q / 2$, our result of the fluctuation analysis is

$$
m^{2}=(2 n-1) q\left(1-\frac{1}{3}\left(2 \pi \alpha^{\prime} q\right)^{2}\right)=\left(n-\frac{1}{2}\right) \frac{\theta}{\pi \alpha^{\prime}}+\mathcal{O}\left(\theta^{5}\right) .
$$

This coincides with the worldsheet result (2.1), up to $\mathcal{O}\left(\theta^{5}\right)$ ambiguity.

If we assume that the action is given by the symmetrized trace at order $F^{6}$, we can soon find that the mass spectrum is incorrect as was shown in Ref. [7]. The non-Abelian BI action at order $F^{6}$ was proposed in some articles [22] and we can use our fluctuation analysis for the first nontrivial check of their $F^{6}$ terms [23].

\section{B. Tachyon mass squared to higher order in $\phi$}

In this appendix, we present a trial to obtain higher order corrections to the tachyon mass eigen values obtained in Section 3. To proceed, we somehow assume the form of the higher order coupling in the tachyon-gauge system of the brane-antibrane. A trial is the following action with the higher correction of gauge fields,

$$
S=-\int d^{3} x\left(-\frac{1}{4 \alpha^{\prime}}|T|^{2}+\frac{1}{2} G^{a b} D_{a} T D_{b} \bar{T}\right),
$$

where $G^{a b}$ is the open string metric

$$
G^{a b}=\left(\frac{1}{\eta+2 \pi \alpha^{\prime} F}\right)_{\mathrm{sym}} .
$$

This fluctuation action can be obtained from the action for example,

$$
S=-2 T \int d x^{3} V(|T|) \sqrt{-\operatorname{det}\left(g_{a b}+F_{a b}+D_{\{a} T D_{b\}} \bar{T}\right)} .
$$

This action is the generalization of a non-BPS D-brane action to the brane-antibrane system. The antisymmetric part of $D_{a} T D_{b} \bar{T}$ is dropped by the reality condition.

Now, let us check the order $O\left(\phi^{2}\right)$ of the mass spectrum in this action (B.1). We proceed in the same manner as in Section 3, to obtain the fluctuation Lagrangian

$$
S=-\int d t d y\left(-\frac{1}{4 \alpha^{\prime}} T^{2}+\frac{1}{2}\left(\partial_{\mu} T\right)^{2}+\frac{\left[\left(\partial_{y} T\right)^{2}+4 \tan ^{2}(\phi / 2) y^{2} T^{2}\right]}{2\left(1+4 \tan ^{2}(\phi / 2)\right)}\right) .
$$

From this the mass squared is obtained as

$$
m^{2}=\frac{\tan (\phi / 2)}{\pi \alpha^{\prime}\left(1+4 \tan ^{2}(\phi / 2)\right)}(2 n+1)-\frac{1}{2 \alpha^{\prime}},
$$

and for the lowest mode, it is given by

$$
\sim \frac{-\theta}{2 \pi \alpha^{\prime}}+O\left((\pi-\theta)^{3}\right) .
$$

Therefore we have obtained the correct mass spectrum up to $O\left((\pi-\theta)^{3}\right)$ ambiguity. 


\section{References}

[1] T. Banks, W. Fischler, S. H. Shenker and L. Susskind, "M Theory As A Matrix Model: A Conjecture," Phys. Rev. D55 (1997) 5112, hep-th/9610043 ;

N. Ishibashi, H. Kawai, Y. Kitazawa and A. Tsuchiya, "A Large-N Reduced Model as Superstring," Nucl. Phys. B498 (1997) 467, hep-th/9612115.

[2] H. Hata, K. Itoh, T. Kugo, H. Kunitomo and K. Ogawa, "Gauge Invariant Action of Interacting String Field," Nucl. Phys. B283 (1987) 433 ; "Covariant String Field Theory," Phys. Rev. D34 (1986) 2360.

[3] C. Bachas, "A way to break supersymmetry," hep-th/9503030;

C. Angelantonj, I. Antoniadis, E. Dudas, A. Sagnotti, "Type-I strings on magnetised orbifolds and brane transmutation," Phys. Lett. B489 (2000) 223, hep-th/0007090;

R. Blumenhagen, L. Görlich, B. Körs and D. Lüst, "Noncommutative Compactifications of Type I Strings on Tori with Magnetic Background Flux," JHEP 0010 (2000) 006, hep-th/0007024;

G. Aldazabal, S. Franco, L. E. Ibanez, R. Rabadan and A. M. Uranga, " $D=4$ Chiral String Compactifications from Intersecting Branes," hep-th/0011073; "Intersecting Brane Worlds," JHEP 0102 (2001) 047, hep-ph/0011132 ;

R. Blumenhagen, B. Körs and D. Lüst, "Type I Strings with F- and B-Flux," JHEP 0102 (2001) 030, hep-th/0012156 ;

L. E. Ibanez, F. Marchesano and R. Rabadan, "Getting just the Standard Model at Intersecting Branes," JHEP 0111 (2001) 002, hep-th/0105155;

R. Blumenhagen, B. Körs, D. Lüst and T. Ott, "The Standard Model from Stable Intersecting Brane World Orbifolds," Nucl. Phys. B616 (2001) 3, hep-th/0107138;

M. Cvetic, G. Shiu and A. M. Uranga, "Three-Family Supersymmetric Standard-like Models from Intersecting Brane Worlds," Phys. Rev. Lett. 87 (2001) 201801, hep-th/0107143 ; "Chiral Four-Dimensional $N=1$ Supersymmetric Type IIA Orientifolds from Intersecting D6-Branes," Nucl. Phys. B615 (2001) 3, hep-th/0107166 ;

D. Cremades, L. E. Ibanez and F. Marchesano, "Intersecting Brane Models of Particle Physics and the Higgs Mechanism," JHEP 0207 (2002) 022, hep-th/0203160;

R. Blumenhagen, V. Braun, B. Körs and D. Lüst, "Orientifolds of K3 and Calabi-Yau Manifolds with Intersecting D-branes," JHEP 0207 (2002) 026, hep-th/0206038;

C. Kokorelis, "Exact Standard model Structures from Intersecting D5-Branes," hep-th/0207234; 
R. Blumenhagen, L. Görlich and T. Ott, "Supersymmetric Intersecting Branes on the Type IIA T6 $/ Z_{4}$ orientifold," JHEP 0301 (2003) 021, hep-th/0211059 ;

A. M. Uranga, "Chiral four-dimensional string compactifications with intersecting D-branes," hep-th/0301032.

[4] C. Herdeiro, S. Hirano and R. Kallosh, "String Theory and Hybrid Inflation/Acceleration," JHEP 0112 (2001) 027, hep-th/0110271;

J. Garcia-Bellido, R. Rabadan and F. Zamora, "Inflationary Scenarios from Branes at Angles," JHEP 0201 (2002) 036, hep-th/0112147 ;

R. Blumenhagen, B. Körs, D. Lüst and T. Ott, "Hybrid Inflation in Intersecting Brane Worlds," Nucl. Phys. B641 (2002) 235, hep-th/0202124;

K. Dasgupta, C. Herdeiro, S. Hirano and R. Kallosh, "D3/D7 Inflationary Model and M-theory," Phys. Rev. D65 (2002) 126002, hep-th/0203019;

N. Jones, H. Stoica and S. -H. Henry Tye, "Brane Interaction as the Origin of Inflation," JHEP 0207 (2002) 051, hep-th/0203163 ;

S. Sarangi and S. -H. Henry Tye, "Cosmic String Production Towards the End of Brane Inflation," Phys. Lett. B536 (2002) 185, hep-th/0204074;

M. Gomez-Reino and I. Zavala, "Recombination of Intersecting D-Branes and Cosmological Inflation," JHEP 0209 (2002) 020, hep-th/0207278 ;

R. Brandenberger, G. Geshnizjani and S. Watson, "On the Initial Conditions for Brane Inflation," hep-th/0302222.

[5] J. Polchinski, "String Theory II," Cambridge University Press (1998).

[6] A. Sen, "Tachyon Condensation on the Brane Anti-Brane System," JHEP 9808 (1998) 012, hep-th/9805170 ; "Descent Relations among Bosonic D-branes," Int.J.Mod.Phys. A14 (1999) 4061, hep-th/9902105; "Non-BPS States and Branes in String Theory," hep-th/9904207 ; "Universality of the Tachyon Potential," JHEP 9912 (1999) 027, hep-th/9911116.

[7] A. Hashimoto and W. Taylor IV, "Fluctuation Spectra of Tilted and Intersecting D-branes from the Born-Infeld Action," Nucl. Phys. B503 (1997) 193, hep-th/9703217.

[8] M. Berkooz, M. R. Douglas and R. G. Leigh, "Branes Intersecting at Angles," Nucl. Phys. B480 (1996) 265, hep-th/9606139.

[9] A. A. Tseytlin, "Vector Field Effective Action in the Open Superstring theory," Nucl. Phys. B276 (1986) 391, Erratum : ibid B291 (1987) 876 ; "On non-abelian generalisation of Born-Infeld action in string theory," Nucl. Phys. B501 (1997) 41, hep-th/9701125;

D. J. Gross and E. Witten, "Superstring Modifications of Einstein's Equations," Nucl. Phys. B277 (1986) 1. 
[10] E. Witten, "Bound States Of Strings And p-Branes," Nucl. Phys. B460 (1996) 335, hep-th/9510135.

[11] H. Arfaei and M. M. Sheikh Jabbari, "Different D-brane Interactions," Phys. Lett. B394 (1997) 288, hep-th/9608167;

M. M. Sheikh Jabbari, "Classification of Different Branes at Angles," Phys. Lett. B420 (1998) 279, hep-th/9710121.

[12] K. Hashimoto, T. Hirayama and S. Moriyama, "Symmetry Origin of Nonlinear Monopole," JHEP 11 (2000) 014, hep-th/0010026.

[13] A. Sen, "Rolling Tachyon," JHEP 0204 (2002) 048, hep-th/0203211; "Tachyon Matter," JHEP 0207 (2002) 065, hep-th/0203265 ; "Field Theory of Tachyon Matter," Mod. Phys. Lett. A17 (2002) 1797, hep-th/0204143.

[14] K. Hashimoto, "Dynamical Decay of Brane-Antibrane and Dielectric Brane," JHEP 0207 (2002) 035, hep-th/0204203.

[15] N. T. Jones and S. -H. Henry Tye, "An Improved Brane Anti-Brane Action from Boundary Superstring Field Theory and Multi-Vortex Solutions," JHEP 0301 (2003) 012, hep-th/0211180.

[16] C. G. Callan, Jr. and J. M. Maldacena, "Brane Dynamics From the Born-Infeld Action," Nucl. Phys. B513 (1998) 198, hep-th/9708147.

[17] A. H. Guth and S. -Y. Pi, "Quantum mechanics of the scalar field in the new inflationary universe," Phys. Rev. D32 (1985) 1899.

[18] A. M. Uranga, "Localized instabilities at conifolds," hep-th/0204079.

[19] M. Gutperle and A. Strominger, JHEP 0204 (2002) 018, hep-th/0202210.

[20] K. Hashimoto, P. -M. Ho and J. E. Wang, "S-brane Actions," to appear in Phys. Rev. Lett., hep-th/0211090;

K. Hashimoto, P. -M. Ho, S. Nagaoka and J. E. Wang, "Time Evolution via S-branes," hep-th/0303172.

[21] A. Vilenkin and E. P. S. Shellard, "Cosmic Strings and Other Topological Defects," Cambridge University Press (1994).

[22] P. Koerber and A. Sevrin, "The non-abelian D-brane effective action through order

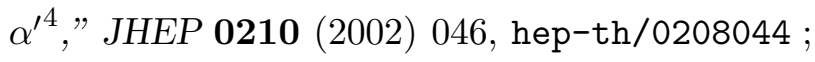

F. Denef, A. Sevrin and J. Troost, "Non-abelian Born-Infeld versus string theory," Nucl. Phys. B581 (2000) 135, hep-th/0002180;

A. Sevrin, J. Troost and W. Troost, "The non-abelian Born-Infeld action at order $F^{6}$," Nucl. Phys. B603 (2001) 389, hep-th/0101192 ; 
A. Bilal, "Higher derivative corrections to the non-abelian Born-Infeld action," Nucl. Phys. B618 (2001) 21, hep-th/0106062.

[23] S. Nagaoka, work in progress.

[24] A. V. Morosov, "Classical Decay of the Non-SUSY-Preserving Configuration of Two D-Branes," Phys. Lett. B433 (1998) 291, hep-th/9803110. 